\title{
Perturbations of Generalized Dual Banach Frames
}

\author{
Seyedeh Sara Karimizad and Mohammad Sadegh Asgari* \\ Department of Mathematics, Faculty of Science, Islamic Azad University, Central Tehran Branch, Tehran, Iran; \\ s_karimizad@yahoo.com, moh.asgari@iauctb.ac. ir, msasgari@yahoo.com,
}

\begin{abstract}
The classical duals and generalized duals of frames play a fundamental role in the abstract frame theory. In this paper we first define the concepts of dual, canonical dual, pseudo-dual and approximate dual for Bessel sequences with respect to a $B K$-space of scalar-valued sequences in Banach spaces, and illuminate their relationship with Banach frames. Then we prove that classical dual and approximate dual of Banach frames are stable under small perturbations so that the results obtained $^{1}$ is a special case of it. We also apply a new characterization of classical dual Banach frames to discuss a stability problem for them. For approximate dual Banach frames constructed via perturbation theory, we provide a bound on the deviation from perfect reconstruction.
\end{abstract}

Keywords: $X_{d}$-Frames, Banach Frames, Approximate Duals, Pseudo-duals, Perturbations.

2010 AMS Subject Classification: Primary 42C99; Secondary 46B99, 46C99.

\section{Introduction}

$\ell^{2}$-Frames in Hilbert spaces were first introduced by Duffin and Schaeffer ${ }^{2}$ in the study of nonharmonic Fourier series in 1952 and then were applied by Daubechies et al. ${ }^{3}$ in the wavelet and Gabor transform. On the other hand, Grochenig ${ }^{4}$ extended $\ell^{p}$-frames to Banach frames with respect to a BK-space of scalar-valued sequences in Ban ach spaces. Further $\ell^{2}$-frames also were introduced by Aldroubi et al. ${ }^{5}$ and Christensen et al. ${ }^{6}$ as a tool to obtain series expansions in shift-invariant spaces. The theory of $\ell^{2}$-frames in Hilbert spaces presents a central tool in many areas and has developed rather rapidly in the past decade. Dual Banach frames pair are sequences in a Banach space and its dual that have basis-like properties but which need not be basis. In particular, they allow elements of a Banach space to be written as linear combinations of the Banach frame elements. Unfortunately, it is usually complicated to calculate a dual Banach frame explicitly. Hence we seek methods for constructing generalized duals. Approximate dual and pseudo-dual frames in Hilbert spaces are defined by Christensen in ${ }^{7}$. The main purpose of this paper is to further and perfect study of the concepts of pseudo-dual and approximate dual Banach frames and examines their properties. We also investigate the use of perturbation theory to construct pseudo-dual and approximate dual Banach frames.

In the rest of this introduction we will briefly recall the definitions and basic properties of Banach frames and bases. For more information we refer to the works of Casazza ${ }^{8}$. Then, in Section 2 we discuss dual Banach frames and find some characterizations about them.

In Section 3 we introduce the concepts of pseudo-dual and approximate dual Banach frames and we show that these concepts are stable under small perturbations. We introduce new and weaker conditions which ensure the desired stability.

Throughout the paper $X$ will be a separable Banach space and $I$ is a countable index set that has been wellordered. We shall denote by $I_{n}, n \in \mathbb{N}$, the subset of the first $n$ indices in $I$. If $|I|<\infty$ then $I_{n}=I$ for all $n \geq|I|$. The notation $X_{d}$ will always be reserved to denote a $B K$-space on $I$.

In a $B K$-space the canonical unit vectors are the elements $e_{i}$ defined by $e_{i}(j)=\delta_{i j}$ where $\delta_{i j}$ is the Kronecker

*Author for correspondence: 
delta. We shall also require that the canonical unit vectors $e_{i}, i \in I$ form a Schauder basis for $X_{d^{*}}$ In other words, every $c \in X_{d}$ equals $\sum_{i \in I} c_{i} e_{i}$ in the sense that $\lim _{n \rightarrow \infty}\left\|c-\sum_{i \in I_{n}} c_{i} e_{i}\right\|_{X_{d}}=0$ and the coefficients in a decomposition of $\mathrm{c}$ into $e_{i}$ are unique. Moreover the dual space $X_{d}^{*}$ of $X_{d}$ is also a $B K$-space of sequences $d=\left\{d_{i}\right\}_{i \in I} \subseteq \mathrm{C}$ such that $d(c)=\sum_{i \in I} c_{i} d_{i}$ for all $c \in X_{d}$. Specifically, we also require that $X_{d}$ be reflexive and the canonical unit vectors $e_{i}, i \in I$ form a Schauder basis for $X_{d}^{*}$ as well, if $d=\left\{d_{i}\right\}_{i \in I} \subseteq \mathrm{C}$ satisfies $\sum_{i \in I} c_{i} d_{i}$ converges for every $d \in X_{d}^{*}$, then $d \in X_{d}^{*}$, and if the above series converges for all $d \in X_{d}^{*}$ then $c \in X_{d}$.

Recall that a family $\left\{f_{i}\right\}_{i \in I} \subseteq X^{*}$ is a $X_{d}$-Bessel sequence for $X$ if $\left\{f_{i}(x)\right\}_{i \in I} \in X_{d}$ for all $x \in X$, it is called a $X_{d}$-frame for $X$ if it is a $X_{d}$-Bessel sequence and there exist $0<A \leq B<\infty$ such that

$$
A\|x\|_{X} \leq\left\|\left\{f_{i}(x)\right\}_{i \in I}\right\|_{X_{d}} \leq B\|x\|_{X} \forall x \in X .
$$

The constants $A$ and $\mathrm{B}$ are called a lower and upper frame bound for $X_{d}$-frame. The analysis operator $U: X \rightarrow X_{d}$ of a $X_{d}$-frame $\left\{f_{i}\right\}_{i \in I}$ is defined by $U_{x}=\left\{f_{i}(x)\right\}_{i \in I}$ which is a bounded operator. (see Proposition $\left.2.3^{9}\right)$. A $X_{d}$-frame $\left\{f_{i}\right\}_{i \in I}$ is called a Banach frame for $X$ with respect to $X_{d}$ if there exists a bounded linear operator $S_{l}: X_{d} \rightarrow X$ such that $S_{l} U_{x}=x$ all $x \in X$. In this case the mapping $S_{l}$ is siad the reconstruction operator of Banach frame and the optimal Banach frame bounds are $\left\|S_{l}\right\|^{-1},\|U\|$.

By replacing $X_{d}$ by $X_{d}^{*}$ and $X$ by $X^{*}$, we can define a $X_{d}^{*}$-frame for $X^{*}$ as an indexed set of elements from $X$ as follows:

DeFinition 1.1: A sequence $\left\{x_{i}\right\}_{i \in I} \subseteq X$ is a $X_{d}^{*}$-frame for $X^{*}$ with frame bounds $0<A \leq B<\infty$ if $\left\{f\left(x_{i}\right)\right\}_{i \in I} X_{d}^{*}$ and

$$
A\|f\|_{X^{*}} \leq\left\|\left\{f\left(x_{i}\right)\right\}_{i \in I}\right\|_{X_{d}} \leq B\|f\|_{X^{*}}
$$

for all $f \in X^{*}$. If $\left\{x_{i}\right\}_{i \in I}$ possesses an upper frame bound, but not necessarily a lower bound, we call it a $X_{d}^{*}$-Bessel sequence with Bessel bound $B$.

The synthesis operator $V: X_{d} \rightarrow X$ of a $X_{d}^{*}$-Bessel sequence $\left\{x_{i}\right\}_{i \in I}$ defined by $V c=\sum_{i \in I} c_{i} x_{i}$, for all $c \in X_{d}$.
As the proof of Proposition $2.3^{9}$ we can prove that $V: X_{d} \rightarrow X$ is also a bounded operator. We call $\left\{x_{i}\right\}_{i \in I}$ a Banach frame for $X^{*}$ with respect to $X_{d}^{*}$. If it is a $X_{d}^{*}$-frame for $X^{*}$ and there exists a bounded linear operator $S_{r}: X \rightarrow X_{d}$ such that $V S_{r} x=x$, for all $x \in X$. Similarly the mapping $S_{r}$ is siad the reconstruction operator and the optimal Banach frame hounds are $\left\|S_{r}\right\|^{-1},\|V\|$.

We say that a family $\left\{f_{i}\right\}_{i \in I} \subset X^{*}$ is total in $X^{*}$, if $f_{i}(x)=0$, for all $i \in I$ then $x=0$. Similarly a sequence $\left\{x_{i}\right\}_{i \in I}$ in $\mathrm{X}$ is said to be total, if $f\left(x_{i}\right)=0$, for all $i \in I$ then $f=0$. The following example shows that in a Hilbert space, there is a Banach frame with respect to a $B K$-space which is not a Banach frame with respect to $\ell^{2}(\mathrm{~N})$.

EXAMPLE 1.2: Suppose that $\left\{e_{i}\right\}_{i=1}^{\infty}$ is an orthonormal basis for a separable Hilbert space $H$. Comisider the family $\left\{e_{i}+e_{i+1}\right\}_{i=1}^{\infty}$ which is a complete set in $H$. Then by Lemma $2.6^{10}$, it is a Banach frame with respect to the $B K$-space

$$
X_{d}=\left\{\left\{\left\langle h, e_{i}+e_{i+1}\right\rangle\right\}_{i=1}^{\infty} \mid h \in H\right\}
$$

but not a Banach frame for $H$ with respect to $\ell^{2}(\mathbb{N})$.

DeFinition 1.3: Let $\left\{x_{i}\right\}_{i \in I}$ be a sequence in $X$. Then

(i) $\left\{x_{i}\right\}_{i \in I}$ is called a Schauder basis for $\mathrm{X}$ if for every $x \in X$ there is an unique sequence of scalars $\left\{c_{i}\right\}_{i \in I}$ which called the coordinates of $x$, such that $x=\sum_{i \in I} c_{i} x_{i}$.

(ii) $\left\{x_{i}\right\}_{i \in I}$ is said to be a $X_{d}$-Riesz basis for $X$ if it is a total set in $X$ and there exist two positive constants $0<A<B<\infty$ such that,

$$
A\|c\|_{X_{d}} \leq\left\|\sum_{i \in I} c_{i} x_{i}\right\|_{X} \leq B\|c\|_{X_{d}} \quad \forall c \in X_{d} .
$$

The constants $A$ and $B$ are called the lower and upper Riesz bounds, respectively. Let $\left\{x_{i}\right\}_{i \in I}$ be a Schauder basis for $\mathrm{X}$, then the linear functionals $f_{i} \in X^{*},(i \in I)$ defined by $f_{i}\left(\sum_{i \in I} c_{i} x_{i}\right)=c_{i}$ is called the coefficient functionals of $\left\{x_{i}\right\}_{i \in I}$ If we define the sequence space $X_{d}$ by

$$
X_{d}=\left\{\left\{f_{i}(x)_{i \in I}\right\} \mid x \in X\right\}
$$

with norm given by $\left\|\left\{f_{i}(x)_{i \in I}\right\}\right\|_{X_{d}}=\|x\|_{X}$, Then $X_{d}$ is a $B K$-Space with the dual space. 


$$
X_{d}^{*}=\left\{\left\{f\left(x_{i}\right)_{i \in I}\right\} \mid f \in X^{*}\right\}
$$

which its norm given by $\left\|\left\{\mathrm{f}\left(\mathrm{x} \_\mathrm{i}\right)\right\}_{\_} \mathrm{i} \in \mathrm{I}\right\|\left\|_{-} \mathrm{Xd} \mathrm{d}^{*}=\right\| \mathrm{f} \| \mathrm{X}^{*}$. The above definitions show that every $x \in X$ has a unique expansion of the form $x=\sum_{i \in I} f_{i}(x) x_{i}$ and $\left\{f_{i}\right\}_{i \in I},\left\{x_{i}\right\}_{i \in I}$ are Banach frames for $X, X^{*}$ with respect to respectively $X_{d}, X_{d}^{*}$ respectively. Since $\left\|\sum_{i \in I} f_{i}(x) x_{i}\right\|_{X}=\|x\|_{X}=\left\|\left\{f_{i}(x)\right\}_{i \in I}\right\|_{X_{d}}$ hence $\left\{x_{i}\right\}_{i \in I}$ is also a $X_{d}$-Riesz basis for $X$.

A sequence $\left\{x_{i}\right\}_{i \in I}$ is called minimal in $X$ if for every $i \in I, x_{i} \notin \operatorname{span}\left\{x_{k}\right\}_{\substack{k \in I \\ k \neq i}}$. The following Propositions are important to characterising $X_{d}^{*}$-frames amid $X_{d}$-Riesz bases which were proved ${ }^{9}$.

Proposition 1.4: Let $\left\{x_{i}\right\}_{i \in I} \subseteq X$ and $\left\{f_{i}\right\}_{i \in I} \subseteq X^{*}$ Then

(i) $\left\{f_{i}\right\}_{i \in I}$ is a $X_{d}$-Bessel sequence for $X$ with $X_{d}$-Bessel bound $B$ if and only if $\sum_{i \in I} d_{i} f_{i}$ converges in $X^{*}$ for all $d \in X_{d}^{*}$ and $\left\|\sum_{i \in I} d_{i} f_{i}\right\|_{X^{*}} \leq B\|d\|_{X_{d}^{*}}$.

(ii) $\left\{x_{i}\right\}_{i \in I}$ is a $X_{d}^{*}$-Bessel sequence for $X^{*}$ with $X_{d}^{*}$-Bessel bound $B$ if and only if $\sum_{i \in I} c_{i} x_{i}$ converges in $X$ for all $c \in X_{d}\left\|\sum_{i \in I} c_{i} x_{i}\right\|_{X^{*}} \leq B\|c\|_{X_{d}^{*}}$

(iii) $\left\{x_{i}\right\}_{i \in I}$ is a $X_{d}^{*}$-frame for $X^{*}$ if and only if, the synthesis operator $V: X_{d} \rightarrow X$, is bounded and possess a bounded inverse on $R_{v}$.

Proposition 1.5: The sequence $\left\{x_{i}\right\}_{i \in I}$ is a $X_{d}$-Riesz basis with Riesz bounds $A, B$ for $X$ if amid only if $\left\{x_{i}\right\}_{i \in I}$ is minimal in $X$ and a $X_{d}^{*}$-frame for $X^{*}$ with same bounds $A, B$.

\section{Dual Banach Frames}

We start this section by definition of dual Banach frames and proving some characterizations about them. Then we discuss a perturbation property for dual Banach frames.

DeFInITION 2.1: Let $\left\{f_{i}\right\}_{i \in I} \subseteq X^{*},\left\{x_{i}\right\}_{i \in I} \subseteq X$ be $X_{d}$-Bessel and $X_{d}^{*}$-Bessel sequences for $X, X^{*}$ respectively. Then

(i) $\left\{f_{i}\right\}_{i \in I}$ is called a dual Banach frame for $\left\{x_{i}\right\}_{i \in I}$ in $X$ with respect to $X_{d}$, if $x=\sum_{i \in I} f_{i}(x) x_{i}$ for all $x \in X$, with the norm convergent sense in $X$. (ii) $\left\{x_{i}\right\}_{i \in I}$ is called a dual Banach frame for $\left\{f_{i}\right\}_{i \in I}$ in $X^{*}$ with respect to $X_{d}^{*}$, if $f=\sum_{i \in I} f\left(x_{i}\right) f_{i}$ for all $f \in X^{*}$, with the norm convergent sense in $X^{*}$.

We begin with a equivalence result of duality on the $X_{d}$-Bessel and $X_{d}^{*}$-Bessel sequences respectively.

LEMMA 2.2: Let $\left\{f_{i}\right\}_{i \in I},\left\{x_{i}\right\}_{i \in I}$ be $X_{d}$-Bessel and $X_{d}^{*}$-Bessel sequences for $X, X^{*}$ respectively.

Then the following statements are equivalent:

(i) $\left\{f_{i}\right\}_{i \in I}$ is a dual Banach frame for $\left\{x_{i}\right\}_{i \in I}$ in $\mathrm{X}$ with respect to $X_{d}$.

(ii) $\left\{x_{i}\right\}_{i \in I}$ is a dual Banach frame for $\left\{f_{i}\right\}_{i \in I}$ in $X^{*}$ with respect to $X_{d}^{*}$.

(iii) $f(x)=\sum_{i \in I} f_{i}(x) f\left(x_{i}\right)$ for all $x \in X, f \in X^{*}$.

Proof. To prove (i) $\Rightarrow$ (ii) let $f \in X^{*}$ be arbitrary then by Proposition 1.4 the series $\Sigma_{i \in I} f\left(x_{i}\right) f_{i}$ is convergent in $X^{*}$ and for every $x \in X$ we have:

$$
f(x)=f\left(\sum_{i \in I} f_{i}(x) x_{i}\right)=\sum_{i \in I} f\left(x_{i}\right) f_{i}(x)=\left(\sum_{i \in I} f_{i}(x) f_{i}\right)(x)
$$

This shows that $f=\Sigma_{i \in I} f\left(x_{i}\right) f_{i}$. The implications (ii) $\Rightarrow$ (iii) and (iii) $\Rightarrow$ (i) are obvious.

Therefore, a pair of $X_{d}$-Bessel and $X_{d^{*}}^{*}$ Bessel sequences $\left\{f_{i}\right\}_{i \in I},\left\{x_{i}\right\}_{i \in I}$ is called a dual Banach frame pair for $X$ or $X^{*}$, if one of the conditions in Lemma 2.2 is satisfied.

LeмmA 2.3: Let $\left\{f_{i}\right\}_{i \in I},\left\{x_{i}\right\}_{i \in I}$ be a dual Banach frame pair for $X$, then $\left\{f_{i}\right\}_{i \in I},\left\{x_{i}\right\}_{i \in I}$ are Banach frames for $X$, $X^{*}$ with respect to $X_{d} X_{d}^{*}$ respectively.

Proof: In terms of the analysis and synthesis operators of $\left\{f_{i}\right\}_{i \in I},\left\{x_{i}\right\}_{i \in I}$. The assumption assures that $U^{*} V^{*}(f)=f$ for all $f \in X^{*}$. This follows that

\section{$\|f\|=\left\|U^{*} V^{*}(f)\right\| \leq\|U\|\left\|V^{*}(f)\right\|=\|U\|\left\|\left\{f\left(x_{i}\right)\right\}_{i \in I}\right\|$}

Now from $V U(x)=x$ for all $x \in X$ implies that $\left\{x_{i}\right\}_{i \in I}$ is a Banach frame for $X^{*}$ with respect to $X_{d}^{*}$. Similarly, we can show that $\left\{f_{i}\right\}_{i \in I}$ is also a Banach frame for $X$ with respect to $X_{d}$.

The following proposition will show that a Banach frame for $X$ with respect to $X_{d}$ plays the same role in Banach frame theory as the dual in the theory of bases.

Proposition 2.4: Let $\left\{f_{i}\right\}_{i \in I}$ be a Banach frame for $X$ with respect to $X_{d}$, then there exists a $X_{d}^{*}$-Bessel sequence 
$\left\{\tilde{x}_{i}\right\}_{i \in I}$ for $X^{*}$ such that $\left\{f_{i}\right\}_{i \in I}$ is a dual Banach frame for $\left\{\tilde{x}_{i}\right\}_{i \in I}$ in $X$ with respect to $X_{d}$.

Proof: Since $\left\{f_{i}\right\}_{i \in I}$ is a Banach frame for $X$ with respect to $X_{d}$ hence there exists a bounded operator $S_{l}: X_{d} \rightarrow X$ such that $S_{l} U=I_{X}$ where, $U: X \rightarrow X_{d}$ is the analysis operator of $\left\{f_{i}\right\}_{i \in I}$. Put $\tilde{x}_{i}=S_{l}\left(e_{i}\right)$ where, $e_{i}, i \in I$ is the Schauder basis of the canonical unit vectors in $X_{d^{*}}$. We first show that $\left\{\tilde{x}_{i}\right\}_{i \in I}$ is a $X_{d^{-}}^{*}$ Bessel sequence for $X^{*}$. Given $c \in X_{d}$ and $m, n \in \mathbb{N}$ with $m>n$.

$$
\begin{aligned}
& \left\|\sum_{i \in I_{m}-I_{n}} c_{i} \tilde{x}_{i}\right\|_{X}=\left\|\sum_{i \in I_{m}-I_{n}} c_{i} S_{l}\left(e_{e}\right)_{i}\right\|_{X} \\
& =\left\|S_{l}\left(\sum_{i \in I_{m}-I_{n}} c_{i} e_{i}\right)\right\|\left\|_{X} \leq\right\| S_{l}\|\| \sum_{i \in I_{m}-I_{n}} c_{i} e_{i} \|_{X_{d}} .
\end{aligned}
$$

Since, $c \in X_{d}$ hence $\Sigma_{i \in I} c_{i} e_{i}$ is convergent, this implies that $\left\{\Sigma_{i \in I_{n}} c_{i} \tilde{x}_{i}\right\}_{n \in N}^{d}$ is a Cauchy sequence in $X$ and therefore convergent. By Proposition 1.4, $\left\{\tilde{x}_{i}\right\}_{i \in I}$ is a $X_{d}^{*}$-Bessel sequence for $X^{*}$. Moreover, for every $x \in X$ we have

$$
x=S_{l} U(x)=S_{l}\left(\sum_{i \in I} f_{i}(x) e_{i}\right)=\sum_{i \in I} f_{i}(x) S_{l}\left(e_{i}\right)=\sum_{i \in I} f_{i}(x) \tilde{x}_{i} .
$$

This shows that $\left\{f_{i}\right\}_{i \in I}$ is a dual Banach frame for $\left\{\tilde{x}_{i}\right\}_{i \in I}$ in $X$ with respect to $X_{d}$.

Via Lemma 2.2 the Banach frame $\left\{\tilde{x}_{i}\right\}_{i \in I}=\left\{S_{l}\left(e_{i}\right)\right\}_{i \in I}$ is said to be the canonical dual Banach frame of $\left\{f_{i}\right\}_{i \in I}$ in $X^{*}$ with respect to $X_{d}^{*}$. We also have a parallel result for Banach frames for $X^{*}$ with respect to $X_{d}^{*}$.

Proposition 2.5: Let $\left\{x_{i}\right\}_{i \in I}$ be a Banach frame for $X^{*}$ with respect to $X_{d}^{*}$ then there exists a $X_{d}$-Bessel sequence $\left\{\tilde{f}_{i}\right\}_{i \in I}$ for $\mathrm{X}$ such that $\left\{x_{i}\right\}_{i \in I}$ is a dual Banach frame for $\left\{\tilde{f}_{i}\right\}_{i \in I}$ in $X^{*}$ with respect to $X_{d}^{*}$.

Proof: By the assumption there exists a bounded operator $S_{r}: X \rightarrow X_{d}$ such that $V S_{r}=I_{X}$ where, $V: X_{d} \rightarrow X$ is the synthesis operator of $\left\{x_{i}\right\}_{i \in I}$. Put $\tilde{f}_{i}=S_{r}^{*}\left(e_{i}\right)$, where $\left\{e_{i}\right\}_{i \in I}$ is the Schauder basis of the canonical unit vectors in $X_{d}^{*}$. Since for all $x \in X$ we have:

$$
\begin{aligned}
\left\|\left\{\tilde{f}_{i}(x)\right\}_{i \in I}\right\|_{X_{d}} & =\left\|\left\{S_{r}^{*}\left(e_{i}\right)(x)\right\}_{i \in I}\right\|_{X_{d}} \\
& =\left\|\left\{e_{i}\left(S_{r}(x)\right)\right\}_{i \in I}\right\|_{X_{d}} \\
& =\left\|S_{r}(x)\right\|_{X_{d}} \leq\left\|S_{r}\right\|\|x\|_{X},
\end{aligned}
$$

hence $\left\{\tilde{f}_{i}(x)\right\}_{i \in I}$ is a $X_{\mathrm{d}}$ - Bessel sequence for $X$. Moreover, for all $f \in X^{*}$ we have

$$
f=S_{r}^{*} V^{*}(f)=S_{r}^{*}\left(\sum_{i \in I} f\left(x_{i}\right) e_{i}\right)=\sum_{i \in I} f\left(x_{i}\right) S_{r}^{*}\left(e_{i}\right)=\sum_{i \in I} f\left(x_{i}\right) \tilde{f}_{i .}
$$

From this the result follows.

The Banach frame $\left\{\tilde{f}_{i}\right\}_{i \in I}=\left\{S_{r}^{*}\left(e_{i}\right)\right\}_{i \in I}$ is called the canonical dual Banach frame of $\left\{x_{i}\right\}_{i \in I}$ in $X$ with respect to $X_{d}$. The next theorem generalizes a result of Christensen ${ }^{11}$ to the situation of dual Banach frames. In this theorem we show that every Banach frame has infinitely many dual Banach frames.

Theorem 2.6: Let $\left\{x_{i}\right\}_{i \in I} \subseteq X$ and $\left\{f_{i}\right\}_{i \in I} \subseteq X^{*}$. Then the following holds:

(i) If $\left\{f_{i}\right\}_{i \in I}$ is a Banach frame for $X$ with respect to $X_{d}$ with the analysis operator $U$. Then the dual Banach frames for $\left\{f_{i}\right\}_{i \in I}$ in $X^{*}$ with respect to $X_{d}^{*}$ are precisely the families $\left\{x_{i}\right\}_{i \in I}=\left\{T_{l} e_{i}\right\}_{i \in I}$, where, $T_{l}: X_{d} \rightarrow X$ is a bounded left-inverse of $U$.

(ii) If $\left\{x_{i}\right\}_{i \in I}$ is a Banach frame for $X^{*}$ with respect to $X_{d}^{*}$ with the synthesis operator $V$. Then the dual Banach frames for $\left\{x_{i}\right\}_{i \in I}$ in $X$ with respect to $X_{d}$ are precisely the families $\left\{f_{i}\right\}_{i \in I}=\left\{T_{r}^{*} e_{i}\right\}_{i \in I}$, where, $T_{r}: X \rightarrow X_{d}$ is a bounded right-inverse of $V$.

Proof: The proof is identical to the proof of Propositions 2.4 and 2.5 .

The next theorem is analogous to Lemma 5.7.3 ${ }^{11}$ to the situation of dual Banach frames.

Theorem 2.7: Let $\left\{f_{i}\right\}_{i \in I},\left\{x_{i}\right\}_{i \in I}$ be Banach frames for $X, X^{*}$ with respect to $X_{d}, X_{d}^{*}$ with the analysis and synthesis operators $U, V$, respectively. Then the following holds:

(i) The bounded left-inverses of $U$ are precisely the operators having the form $S_{l}+W\left(I_{X_{d}}-U S_{l}\right)$ where, $W: X \rightarrow$ $X_{d}$ is a bounded operator and $S_{l}$ denotes the reconstruction operator of $\left\{f_{i}\right\}_{i \in I}$.

(ii) The bounded right-inverses of $V$ are precisely the operators having the form $S_{r}+\left(I_{X_{d}}-S_{r} V\right) W$, where $W: X \rightarrow X_{d}$ is a bounded operator and $S_{r}$ denotes time reconstruction operator of $\left\{x_{i}\right\}_{i \in I^{*}}$.

Proof: For the proof of (i), it is obvious that an operator of the given form is a left-inverse of $\mathrm{U}$. On the other hand, if $T_{l}$ is a given left-inverse of $U$, then by taking $W=T_{l}$ we have $T_{l}=S_{l}+T_{l}\left(I_{X_{d}}-U S_{l}\right)$ 
the argument for statement (ii) is similar.

The next theorem is analogous to a well-known result in abstract frame theory Theorem 5.7.4 ${ }^{11}$. This theorem is a characterization of all dual Banach frames associated with a given Banach frame.

Theorem 2.8: Let $\left\{f_{i}\right\}_{i \in I},\left\{x_{i}\right\}_{i \in I}$ be Banach frames for $X$, $X^{*}$ with respect to $X_{d}, X_{d}^{*}$ with the analysis and synthesis operators $U, V$, respectively. Then the following holds:

(i) The dual Banach frames of $\left\{f_{i}\right\}_{i \in I}$ in $X^{*}$ with respect to $X_{d}^{*}$ are precisely the families

$$
\left\{z_{k}\right\}_{k \in I}=\left\{\tilde{x}_{k}+y_{k}-\sum_{i \in I} f_{i}\left(\tilde{x}_{k}\right) y_{i}\right\}_{k \in I},
$$

where, $\left\{y_{k}\right\}_{k \in I}$ is a $X_{d}^{*}$-Bassel sequence for $\mathrm{X}^{*}$ and $\left\{x_{i}\right\}_{i \in I}$ denotes the canonical dual Banach frame of $\left\{f_{i}\right\}_{i \in I}$ in $X^{*}$ with respect to $X_{d}^{*}$.

(ii) The dual Banach frames of $\left\{x_{i}\right\}_{i \in I}$ in $X$ with respect to $X_{d}$ are precisciy the families

$$
\left\{g_{k}\right\}_{k \in I}=\left\{\tilde{f}_{k}+h_{k}-\sum_{i \in I} \tilde{f}_{k}\left(x_{i}\right) h_{i}\right\}_{k \in I},
$$

where, $\left\{h_{k}\right\}_{k \in I}$ is a $X_{d}$-Bessel sequence for $\mathrm{X}$ and $\left\{\tilde{f}_{i}\right\}_{i \in I}$ denotes the canonical dual Banach frame of $\left\{x_{i}\right\}_{i \in I}$ in $\mathrm{X}$ with respect to $X_{d}$.

Proof: (i) By Theorem 2.6 and Theorem 2.7, we can characterize the dual Banach frames of $\left\{f_{i}\right\}_{i \in I}$ in $X^{*}$ with respect to $X_{d}^{*}$ as all families of the form $\left\{z_{k}\right\}_{k \in I}=\left\{\left(S_{l}+W\left(I_{X_{d}}-U S_{l}\right) e_{k}\right)\right\}_{k \in I}$, where $W: X_{d} \rightarrow X$ is a bounded operator, or equivalently an operator of the form $W(c)=\Sigma_{i \in I} c_{i} y_{i}$ where $\left\{y_{k}\right\}_{k \in I}$ is a $X_{d}^{*}$-Bessel sequence for $X^{*}$, Inserting this expression for $W$ we obtain.

$$
\begin{aligned}
\left\{z_{k}\right\}_{k \in I} & =\left\{S_{l}\left(e_{k}\right)+W\left(e_{k}\right)-W U S_{l}\left(e_{k}\right)\right\}_{k \in I} \\
& =\left\{\tilde{x}_{k}+y_{k}-\sum_{i \in I} f_{i}\left(\tilde{x}_{k}\right) y_{i}\right\}_{k \in I} .
\end{aligned}
$$

The proof for the statement (ii) is analogous.

Definition 2.9: A nonzero operator $\Lambda \in B(X, Y)$ is called a left divisor of zero if there exists a nonzero operator $\Gamma \in B(Y, X)$ such that $\Lambda \Gamma=0$, similarly a nonzero operator $\Lambda \in B(X, Y)$ is called a right divisor of zero if there exists a nonzero operator $\Gamma \in B(Y, X)$ such that $\Gamma \Lambda=0$.
LEMMA 2.10: Let $\left\{f_{i}\right\}_{i \in I},\left\{x_{i}\right\}_{i \in I}$ be Banach frames for $X$, $X^{*}$ with respect to $X_{d}, X_{d}^{*}$, then the analysis and synthesis operators of them are right and left divisors of zero in $B\left(X, X_{d}\right), B\left(X_{d}, X\right)$ respectively.

Proof: Suppose that $U, V$ are the analysis and synthesis operators of $\left\{f_{i}\right\}_{i \in I},\left\{x_{i}\right\}_{i \in I}$ respectively.

Letting $\Lambda: X_{d} \rightarrow X$ and $\Gamma: X \rightarrow X_{d}$ by $\Lambda=I_{X}-U S_{l}$ and $\Gamma=I_{X}-S_{r} V$, where $S_{p}, S_{r}$ denote the reconstruction operators of $\left\{f_{i}\right\}_{i \in I},\left\{x_{i}\right\}_{i \in I}$ Then we have $\Lambda U=0$ and $V \Gamma=0$.

Time following Theorem is another characterization from the dual Banach frames by the family of left and right divisors of zero.

TheOREM 2.11: Let $\left\{f_{i}\right\}_{i \in I},\left\{x_{i}\right\}_{i \in I}$ be Banach frames for $X, X^{*}$ with respect to $X_{d}, X_{d}^{*}$ with the analysis and synthesis operators $U, V$, respectively. Then the following holds:

(i) There exists an one to one correspondence between dual Banach frames of $\left\{f_{i}\right\}_{i \in I}$ in $X^{*}$ with respect to $X_{d}^{*}$ and the bounded operators $\Lambda: X_{d} \rightarrow X$ such that $\Lambda \mathrm{U}=0$.

(ii) There exists an one to one correspondence between dual Banach frames of $\left\{x_{i}\right\}_{i \in I}$ in $X$ with respect to $X_{d}$ and the bounded operators $\Gamma: X \rightarrow X_{d}$ such that $V \Gamma=0$.

Proof: (i) Let $\left\{y_{i}\right\}_{i \in I}$ be a dual Banach frame of $\left\{f_{i}\right\}_{i \in I}$ in $X^{*}$ with respect to $X_{d}^{*}$ with the synthesis operator $W$. Define $\Lambda: X_{d} \rightarrow X$ by $\Lambda=W-S_{l}$. where, $S_{l}$ denotes the reconstruction operator of $\left\{f_{i}\right\}_{i \in I}$ Clearly, $\Lambda$ is a bounded operator and by using Lemma 2.2 we have.

$$
\Lambda \mathrm{U}=\mathrm{WU}-S_{l} \mathrm{U}=0
$$

For the opposite implication, suppose that $\Lambda$ is a bounded operator from $X_{d}$ in $X$ such that $\Lambda \mathrm{U}=0$. Letting $y_{i}=S_{l} e_{i}+\Lambda e_{i}, i \in I_{\text {where }}\left\{e_{i}\right\}_{i \in I}$ denotesthe Schauder basis of the canonicalunitvectorsin $X_{d}$. AstheproofofProposition $2.4,\left\{y_{i}\right\}_{i \in I}$ is a $X_{d}^{*}$-Bessel sequence for $X^{*}$ and for every $x \in X$ we have

$$
\sum_{i \in I} f_{i}(x) y_{i}=\sum_{i \in I} f_{i}(x) S_{l} e_{i}+\sum_{i \in I} f_{i}(x) \Lambda e_{i}=S_{l} U x+\Lambda U x=x .
$$

This shows that $\left\{y_{i}\right\}_{i \in I}$ is a dual Banach frame of $\left\{f_{i}\right\}_{i \in I}$ in $X^{*}$ with respect to $X_{d}^{*}$. 
(ii) The proof is similar to (i).

The following theorem is a perturbation result of dual Banach frames.

Theorem 2.12: Let $\left\{f_{i}\right\}_{i \in I},\left\{f_{i}^{\prime}\right\}_{i \in I}$ be Banach frames for $\mathrm{X}$ with respect to $X_{d}$ and let $\left\{x_{i}\right\}_{i \in I}$ be a dual Banach frame of $\left\{f_{i}\right\}_{i \in I}$ in $X^{*}$ with respect to $X_{d}^{*}$. If $\left\{f_{i}-f_{i}^{\prime}\right\}_{i \in I}$ and $\left\{\tilde{x}_{i}-\tilde{x}_{i}^{\prime}\right\}_{i \in I}$ are two $X_{d}$-Bessel $X_{d}^{*}$ - Bessel sequences for $X, X^{*}$ with sufficiently small Bessel bounds $\varepsilon$. Then there exists a dual Banach frame $\left\{x_{i}^{\prime}\right\}_{i \in I}$ for $\left\{f_{i}\right\}_{i \in I}$ in $X^{*}$ with respect to $X_{d}^{*}$ such that $\left\{x_{i}-x_{i}^{\prime}\right\}_{i \in I}$ is a $X_{d}^{*}$-Bessel sequence in $X^{*}$ and its bound is a multiple of $\varepsilon$.

Proof: Suppose that U, $S_{l}$ and $U^{\prime}, S_{l}^{\prime}$ are the analysis and reconstruction operators of $\left\{f_{i}\right\}_{i \in I},\left\{f_{i}^{\prime}\right\}_{i \in I}$, then $\tilde{x}_{i}=S_{l} e_{i}, \tilde{x}_{i}^{\prime}=S_{l}^{\prime} e_{i}$ for all $i \in I$, where $\left\{e_{i}\right\}_{i \in I}$ denotes the Schauder basis of the canonical unit vectors in $X_{d}$. By the Theorem 2.11 there exists a bounded operator $\Lambda: X_{d} \rightarrow X$ such that $\Lambda U=0$ and $x_{i}=\tilde{x}_{i}+\Lambda e_{i}, i \in I$. Defining $y_{i}=\tilde{x}_{i}^{\prime}+\Lambda e_{i}$, it is easy to check that $\left\{y_{i}\right\}_{i \in I}$ is a $X_{d}^{*}$-Bessel sequence in $X^{*}$. Denoting the synthesis operator of $\left\{y_{i}\right\}_{i \in I}$ by $V$, we claim that the bounded operator $\Gamma x=V U^{\prime} x=\Sigma_{i \in I} f_{i}^{\prime}(x) y_{i}$ is invertible. In fact, for any $x \in X$, we have

$\|x-\Gamma x\|=\left\|x-\sum_{i \in I} f_{i}^{\prime}(x) y_{i}\right\|=\left\|\Lambda U^{\prime} x\right\|=\left\|\Lambda U^{\prime} x-\Lambda U_{x}\right\|$

$\leq\|\Lambda\|\left\|U^{\prime} x-U x\right\| \leq \varepsilon\|\Lambda\|\|x\|$.

Therefore, if $\varepsilon\|\Lambda\|<1$, then $\Gamma$ is invertible and we obtain

$$
\begin{aligned}
& \left\|\Gamma^{-1}\right\| \leq \frac{1}{1-\left\|I_{X}-r\right\|}<\frac{1}{1-\varepsilon\|\Lambda\|} \text { and } \\
& \left\|I_{X}-\Gamma^{-1}\right\|<\frac{\varepsilon}{1-\varepsilon\|\Lambda\|}
\end{aligned}
$$

Put $x_{i}^{\prime}=\Gamma^{-1} y_{i}$ for all $i \in I$ it is trivial that $\left\{x_{i}^{\prime}\right\}_{i \in I}$ is a $X_{d}^{*}$ Bessel sequence in $X^{*}$ and we see from $\Gamma x=\Sigma_{i \in I} f_{i}^{\prime}(x) y_{i}$ that $x=\Sigma_{i \in I} f_{i}^{\prime}(x) x_{i}^{\prime}$. Hence $\left\{x_{i}^{\prime}\right\}_{i \in I}$ is a dual Banach frame for $\left\{f_{i}^{\prime}\right\}_{i \in I}$ in $X^{*}$ with respect to $X_{d}^{*}$.

On the other hand, for all $c \in X_{d}$ we have:

$$
\left\|\sum_{i \in I} c_{i}\left(x_{i}-x_{i}^{\prime}\right)\right\|_{X}=\left\|\sum_{i \in I} C_{i}\left(x_{i}-\Gamma^{-1} x_{i}+\Gamma^{-1} x_{i}-\Gamma^{-1} y_{i}\right)\right\|_{X}
$$

$$
\begin{aligned}
& \leq\left\|I_{X}-\Gamma^{-1}\right\|\left\|\sum_{i \in I} c_{i} x_{i}\right\|_{X}+\left\|\Gamma^{-1}\right\|\left\|\sum_{i \in I} c_{i}\left(\tilde{x}_{i}-\tilde{y}_{i}^{\prime}\right)\right\|_{X} \\
& \leq \frac{\varepsilon}{1-\varepsilon\|\Lambda\|}\left\|\sum_{i \in I} c_{i} x_{i}\right\|_{X}+\frac{1}{1-\varepsilon\|\Lambda\|}\left\|\sum_{i \in I} c_{i}\left(x_{i}-y_{i}\right)\right\|_{X} \\
& \leq \frac{\left\|S_{l}+\Lambda\right\|}{1-\varepsilon\|\Lambda\|}\|c\|_{X_{d}} \varepsilon .
\end{aligned}
$$

This completes the proof.

Corollary 2.13: Let $\left\{x_{i}\right\}_{i \in I},\left\{x_{i}^{\prime}\right\}_{i \in I}$ be Banach frames for $X^{*}$ with respect to $X_{d}^{*}$ and let $\left\{f_{i}\right\}_{i \in I}$ be a dual Banach frame of $\left\{x_{i}\right\}_{i \in I}$ in $X$ with respect to $X_{d}$. If $\left\{\tilde{f}_{i}-\tilde{f}_{i}^{\prime}\right\}_{i \in I}$ and $\left\{x_{i}-x_{i}^{\prime}\right\}_{i \in I}$ are two $X_{d}$-Bessel $X_{d}^{*}$-Bessel sequences for $X, X^{*}$ with sufficiently small Bessel bounds $\varepsilon$. Then there exists a dual Banach frame $\left\{f_{i}^{\prime}\right\}_{i \in I}$ for $\left\{x_{i}^{\prime}\right\}_{i \in I}$ in $X$ with respect to $X_{d}$ such that $\left\{f_{i}-f_{i}^{\prime}\right\}_{i \in I}$ is a $X_{d}$-Bessel sequence in $X$ and its bound is a multiple of $\varepsilon$.

Proof: The proof is similar to Theorem 2.12.

\section{Generalized Dual Banach Frames and Perturbation Results}

In order to apply the dual frame expansions Hilbert space Christensen and Laugesen ${ }^{7}$, introduced the concepts of pseudo-duals and approximate duals for frames in Hilbert spaces. In this section we generalize this concepts in Banach spaces and examines their properties.

Definition 3.1: Suppose that $\left\{f_{i}\right\}_{i \in I},\left\{x_{i}\right\}_{i \in I}$, are $X_{d}$-Bessel and $X_{d}^{*}$-Bessel sequences for $X, X^{*}$ with analysis and synthesis operators $U, V$ respectively. Then

(i) $\left\{f_{i}\right\}_{i \in I}$ is a pseudo-dual Banach frame for $\left\{x_{i}\right\}_{i \in I}$ in $X$ with respect to $X_{d}$, if $V U$ is a bijection on $\mathrm{X}$.

(ii) We say that $\left\{f_{i}\right\}_{i \in I}$ is an approximate dual Banach frame for $\left\{x_{i}\right\}_{i \in I}$ in $X$ with respect to $X_{d}$, If $\left\|I_{X}-V U\right\|<1$.

Note that if $\left\{f_{i}\right\}_{i \in I}$ is a pseudo-dual Banach frame for $\left\{x_{i}\right\}_{i \in I}$ in $X$ with respect to $X_{d}$, then

$$
x=\sum_{i \in I} f_{i}(x)(V U)^{-1} x_{i} \quad \forall x \in X
$$

Thus $\left\{f_{i}\right\}_{i \in I}$ is a dual Banach frame for $\left\{(V U)^{-1} x_{i}\right\}_{i \in I}$ in $X$ with respect to $X_{d}$. From here, a standard argument shows that $\left\{f_{i}\right\}_{i \in I}$ is a Banach frame for $X$ with respect 
to $X_{d^{*}}$ By symmetry $\left\{x_{i}\right\}_{i \in I}$ is also a Banach frame for $X^{*}$ with respect to $X_{d}^{*}$. Furthermore, if $\left\{f_{i}\right\}_{i \in I}$ is an approximate dual Banach frame for $\left\{x_{i}\right\}_{i \in I}$ in $X$ with respect to $X_{d}$. Since the condition $\left\|I_{X}-V U\right\|<1$ implies that the operator $V U$ is a bijection on $X$. Thins every approximate dual Banach frame is a pseudo-dual Banach frame.

The next lemma follows immediately from the definition. We leave the proof to interested readers.

LemMA 3.2: Let $\left\{f_{i}\right\}_{i \in I},\left\{x_{i}\right\}_{i \in I}$ be $X_{d}$-Bessel and $X_{d}^{*}$-Bessel sequences for $X, X^{*}$ with analysis and synthesis operators $U, V$ respectively. Then the following statements are equivalent:

(i) $\left\{f_{i}\right\}_{i \in I}$ is a pseudo-dual Banach frame for $\left\{x_{i}\right\}_{i \in I}$ in $X$ with respect to $X_{d^{*}}$.

(ii) $x=\sum_{i \in I} f_{i}\left((V U)^{-1} x\right) f_{i}=\sum_{i \in I} f_{i}(x)(V U)^{-1} x_{i} \quad \forall x \in X$.

(iii) $f=\sum_{i \in I} f_{i}\left((V U)^{-1} x\right) x_{i}=\sum_{i \in I} f_{i}(x)\left(V^{*} U^{*}\right)^{-1} f_{i} \forall x \in X^{*}$.

(iv) $f(x)=\sum_{i \in I} f\left((V U)^{-1} x\right) f_{i}(x)=\sum_{i \in I} f\left(x_{i}\right) f_{i}\left((V U)^{-1} x_{i}\right.$. $\forall x \in X, f \in X^{*}$

The next theorem shows that approximate dual Banach frames are stable under small perturbations of the Banach frame elements so that Theorem 2.2 obtained in ${ }^{1}$ is a special case of it.

Theorem 3.3: Let $\left\{f_{i}\right\}_{i \in I}$ be a Banach frame for $X$ with respect to $X_{d}$ with the analysis and reconstruction operators $U$, S. Assume that $\left\{g_{i}\right\}_{i \in I} \subseteq X^{*}$ and there exist $\lambda, \mu \geq 0$ such that

(i) $2(\lambda\|U\|+\mu)\|S\|<1$.

(ii) $\left\|\left\{f_{i}(x)-g_{i}(x)\right\}_{i \in I}\right\|_{X_{d}} \leq \lambda\left\|\left\{f_{i}(x)\right\}_{i \in I}\right\|_{X_{d}}+\mu\|x\|_{X}$,

for all $x \in X$. Then $\left\{g_{i}\right\}_{i \in I}$ is a Banach frame for $X$ with respect to $X_{d}$ and $\left\{g_{i}\right\}_{i \in I},\left\{f_{i}\right\}_{i \in I}$ are approximate dual Banach frames of $\left\{\tilde{x}_{i}\right\}_{i \in I},\left\{\tilde{y}_{i}\right\}_{i \in I}$ in $X$ with respect to $X_{d}$ respectively, where $\left\{\tilde{x}_{i}\right\}_{i \in I},\left\{\tilde{y}_{i}\right\}_{i \in I}$ are the canonical dual Banach frame of $\left\{f_{i}\right\}_{i \in I},\left\{g_{i}\right\}_{i \in I}$ in $X^{*}$ with respect to $X_{d}^{*}$.

Proof: Let $U_{1}$ be the analysis operator of $\left\{g_{i}\right\}_{i \in I}$, from the hypotheses we have

$$
\begin{aligned}
& \left\|U_{1} x\right\|_{X_{d}} \leq\left\|U_{1} x-U x\right\|_{X_{d}}+\|U x\|_{X_{d}} \\
& \leq((\lambda+1)\|U\|+\mu)\|x\|_{X},
\end{aligned}
$$

for all $x \in X$. This estabhisbmes the upper frame bound for $\left\{g_{i}\right\}_{i \in I .}$. On the other hand, from $S U=I_{X}$ we have $\left\|I_{X}-S U_{1}\right\| \leq\|S\|\left\|U-U_{1}\right\|<1$ which follows that $\left\{g_{i}\right\}_{i \in I}$ is an approximate dual Banach frame of $\left\{\tilde{x}_{i}\right\}_{i \in I}$ and so $\left\|S_{1}\right\| \leq \frac{1}{1-(\lambda\|U\|+\mu)\|S\|}$.

If we set $S_{1}=\left(S U_{1}\right)^{-1} S$, then $S_{1} U_{1}=I_{X}$ which implies that $\left\{g_{i}\right\}_{i \in I}$ is a Banach frame for $X$ with respect to $X_{d}$ and $\left\|S_{1}\right\| \leq \frac{\|S\|}{1-(\lambda\|U\|+\mu)\|S\|} \quad$ Finally, from $\tilde{y}_{i}=S_{1}\left(e_{i}\right)$ we obtain

$$
\begin{aligned}
& \left\|I_{X}-S_{1} U\right\|=\left\|S_{1} U_{1}-S_{1} U\right\| \leq\left\|S_{1}\right\|\left\|U_{1}-U\right\| \\
& \leq \frac{(\lambda\|U\|+\mu)\|S\|}{1-(\lambda\|U\|+\mu) \|}<1 .
\end{aligned}
$$

This completes the proof.

Corollary 3.4: Let $\left\{f_{i}\right\}_{i \in I}$ be a dual Banach frame of $\left\{x_{i}\right\}_{i \in I}$ in $X$ with respect to $X_{d}$ with the analysis and synthesis operators $U, V$. Assume that $\left\{g_{i}\right\}_{i \in I}$ is a sequence $X^{*}$ in $\mathrm{X}^{*}$ and there exist $\lambda, \mu \geq 0$ such that

(i) $2(\lambda\|U\|+\mu)\|V\|<1$.

(ii) $\left\|\left\{f_{i}(x)-g_{i}(x)\right\}_{i \in I}\right\|_{X_{d}} \leq \lambda\left\|\left\{f_{i}(x)\right\}_{i \in I}\right\|_{X_{d}}+\mu\|x\|_{X}$.

Then $\left\{g_{i}\right\}_{i \in I}$ is an approximate dual Banach frame for $\left\{x_{i}\right\}_{i \in I}$ in $X$ with respect to $X_{d^{*}}$

Proof: The proof is similar to Theorem 3.3.

Theorem 3.5: Let $\left\{f_{i}\right\}_{i \in I}$ be a dual Banach frame for $\left\{x_{i}\right\}_{i \in I}$ in $X$ with respect to $X_{d}$ with the analysis and synthesis operators $U, V$. Assume that $\left\{y_{i}\right\}_{i \in I}$ is a sequence in $X$ and there exist $\lambda, \mu \geq 0$ such that

(i) $(1+\|V\|\|U\|)(\lambda+\mu\|U\|)<1$.

(ii) $\left\|\sum_{i \in I} c_{i}\left(x_{i}-y_{i}\right)\right\|_{X} \leq \lambda\left\|\sum_{i \in I} c_{i} x_{i}\right\|_{X}+\mu\|c\|_{X_{d}}$

for all $c \in X_{d}$. Then $\left\{f_{i}\right\}_{i \in I}$ is an approximate dual Banach frame for $\left\{y_{i}\right\}_{i \in I}$ in $X$ with respect to $X_{d}$. Furthermore, there exists a $X_{d}$-Bessel sequence $\left\{g_{i}\right\}_{i \in I}$ for $X$ such that $\left\{g_{i}\right\}_{i \in I}$ is an approximate dual Banach frame for $\left\{x_{i}\right\}_{i \in I}$ in $X$ with respect to $X_{d}$. 
Proof: The hypotheses given imply that the series $\sum_{i \in I} f_{i}(x) y_{i}$ is convergent in $X$ for every $x \in X$. Thus the operator $\Lambda: X \rightarrow X$ defined by $\Lambda x=\sum_{i \in I} f_{i}(x) y_{i}$ is bounded and holds

$$
\|x-\Lambda x\|_{X} \leq \lambda\|x\|_{X}+\mu\|U x\|_{X_{d}} \leq(\lambda+\mu\|U\|)\|x\|_{X} .
$$

From this we have $\left\|I_{X}-\Lambda\right\|<1$, which follows that is an approximate dual Banach frame for $\left\{y_{i}\right\}_{i \in I}$ in $X$ with respect to $X_{d}$ and $\left\|\Lambda^{-1}\right\| \leq \frac{1}{1-(\lambda+\mu\|U\|)}$. Therefore if we define $g_{i}=\left(\Lambda^{-1}\right)^{*}\left(f_{i}\right), i \in I$, then $\left\{g_{i}\right\}_{i \in I}$ is a dual Banach frame for $\left\{y_{i}\right\}_{i \in I}$ in $X$ with respect to $X_{d}$. Let $U_{1}$ be the analysis operator of $\left\{g_{i}\right\}_{i \in I}$. Then we have

$$
\begin{gathered}
\left\|I_{X}-V U_{1}\right\| \leq\|V\|\left\|U-U_{1}\right\| \leq\|V\|\|U\|\left\|I_{X}-\Lambda^{-1}\right\| \\
\leq\|V\|\|U\|\left\|\Lambda^{-1}\right\|\left\|I_{X}-\Lambda\right\| \leq\|V\|\|U\| \\
\frac{\lambda+\mu\|U\|}{1-(\lambda+\mu\|U\|)}<1 .
\end{gathered}
$$

Therefore $\left\{g_{i}\right\}_{i \in I}$ is an approximate dual Banach frame for $\left\{x_{i}\right\}_{i \in I}$ in $\mathrm{X}$ with respect to $X_{d}$.

Corollary 3.6: Let $\left\{f_{i}\right\}_{i \in I}$ be a dual Banach frame for $\left\{x_{i}\right\}_{i \in I}$ in $X$ with respect to $X_{d}$ with the analysis and synthesis operators $U, V$. Assume that $\left\{y_{i}\right\}_{i \in I}$ is a sequence in $X$ such that

$$
\begin{aligned}
& \left\{\left\|x_{i}-y_{i}\right\|\right\}_{i \in I} \in X_{d}^{*} \text { and }(1+\|V\|\|U\|)\|U\| \| \\
& \left\{\left\|x_{i}-y_{i}\right\|\right\}_{i \in I} \|_{X_{d}^{*}<1}
\end{aligned}
$$

Then $\left\{f_{i}\right\}_{i \in I}$ is an approximate dual Banach frame for $\left\{y_{i}\right\}_{i \in I}$ in $X$ with respect to $X_{d}$ and there exists a $X_{d}$-Bessel sequence $\left\{g_{i}\right\}_{i \in I}$ for $X$ such that $\left\{g_{i}\right\}_{i \in I}$ is an approximate dual Banach frame for $\left\{x_{i}\right\}_{i \in I}$ in $\mathrm{X}$ with respect to $X_{d^{*}}$

Proof: Since for every $c \in X_{d}$ we have

$$
\left\|\sum_{i \in I} c_{i}\left(x_{i}-y_{i}\right)\right\|_{X} \leq\left\|\left\{\left\|x_{i}-y_{i}\right\|\right\}_{i \in I}\right\|_{X_{d}^{*}}\|c\|_{X_{d}}
$$

Therefore the result follows from the Theorem 3.5 with $\lambda=0$ and $\mu=\left\|\left\{\left\|x_{i}-y_{i}\right\|\right\}_{i \in I}\right\|_{X_{d}^{*}}$.

A $B K$-space $X_{d}$ is solid if whenever $\left\{b_{i}\right\}_{i \in I},\left\{c_{i}\right\}_{i \in I}$ are sequences with $\left\{c_{i}\right\}_{i \in I} \in X_{d}$ and $\left|b_{i}\right| \leq\left|c_{i}\right|$, then it follows that $\left\{b_{i}\right\}_{i \in I} \in X_{d}$ and $\left\|\left\{b_{i}\right\}_{i \in I}\right\|_{X_{d}} \leq\left\|\left\{c_{i}\right\}_{i \in I}\right\|_{X d}$. Note that if the canonical unit vectors $e_{i}, i \in I$ form a Schauder basis for $X_{d}$ then $X_{d}$ is solid.

COROLlaRY 3.7: Let $\left\{f_{i}\right\}_{i \in I}$ be a dual Banach frame for $\left\{x_{i}\right\}_{i \in I}$ in $X$ with respect to $X_{d}$ within the analysis and synthesis operators $U, V$. Assume that there exists a family $\left\{\Lambda_{j}\right\}_{j \in J}$ of bounded operators on $X$ and scalars $\alpha_{i j}$ so that $y_{i}=x_{i}-\sum_{j \in J} \alpha_{i j} \Lambda_{j} x_{i}$ for all $i \in I$. If $\alpha_{j}=\operatorname{Sup}\left|\alpha_{i j}\right|<\infty$ for all $j \in J$ and $(1+\|V\|\|U\|)\|V\|\|U\| \sum_{j \in J}^{i \in I} \alpha_{j}\left\|\Lambda_{j}\right\|<1$. Then $\left\{f_{i}\right\}_{i \in I}$ is an approximate dual Banach frame for $\left\{y_{i}\right\}_{i \in I}$ in $X$ with respect to $X_{d}$ and there exists a $X_{d}$-Bessel sequence $\left\{g_{i}\right\}_{i \in I}$ for $\mathrm{X}$ such that $\left\{g_{i}\right\}_{i \in I}$ is an approximate dual Banach frame for $\left\{x_{i}\right\}_{i \in I}$ in $X$ with respect to $X_{d}$.

Proof: For all $j \in J$ and $c \in X_{d}$ we have

$$
\begin{aligned}
& \left\|\sum_{i \in I} c_{i} \alpha_{i j} x_{i}\right\|_{X}=\sup _{\|f\|_{X_{d}^{*}}=1}\left|\sum_{i \in I} c_{i} \alpha_{i j} f\left(x_{i}\right)\right| \\
& \leq \sup _{\|f\|_{X_{d}^{*}=1}^{*}}\left\|V^{*}(f)\right\|_{X_{d}^{*}}\left\|\left\{\alpha_{i j} c_{i}\right\}_{i \in I}\right\|_{X d} \\
& \leq \alpha_{j}\|V\|\|c\|_{X_{d}^{*}}
\end{aligned}
$$

This yields

$$
\begin{aligned}
& \left\|\sum_{i \in I} c_{i}\left(x_{i}-y_{i}\right)\right\|_{X}=\left\|\sum_{i \in I} \sum_{j \in J} c_{i} \alpha_{i j} \Lambda_{j} x_{i}\right\|_{X} \\
& =\left\|\sum_{j \in J} \Lambda_{j}\left(\sum_{i \in I} c_{i} \alpha_{i j} x_{i}\right)\right\|_{X} \\
& \leq \sum_{j \in J}\left\|\Lambda_{j}\right\|\left\|\sum_{i \in I} c_{i} \alpha_{i j} x_{i}\right\|_{X} \\
& \leq\|c\|_{X_{d}}\|V\| \sum_{j \in J} \alpha_{j}\left\|\Lambda_{j}\right\| .
\end{aligned}
$$

Now the result follows from the Theorem 3.5 with $\lambda=0$ and $\mu=\|V\| \sum_{j \in J} \alpha_{j}\left\|\Lambda_{j}\right\|$.

Proposition 3.8: Let $\left\{f_{i}\right\}_{i \in I}$ be an approximate dual Banach frame for $\left\{x_{i}\right\}_{i \in I}$ in $X$ with respect to $X_{d}$ with the analysis and synthesis operators $U, V$, respectively. Then the following holds:

(i) $\left\{\left(U^{*} V^{*}\right)^{-1} f_{i}\right\}_{i \in I}$ is a dual Banach frame for $\left\{x_{i}\right\}_{i \in I}$ in $\mathrm{X}$ with respect to $X_{d}$ and

$$
\left(U^{*} V^{*}\right)^{-1} f_{i}=f_{i}+\sum_{n=1}^{\infty}\left(I_{X^{*}}-U^{*} V^{*}\right)^{n} f_{i}
$$

(ii) For fixed $n \in N$, consider the partial sum $f_{n i}=f_{i}+\sum_{j=1}^{n}\left(I_{X^{*}}-U^{*} V^{*}\right)^{j} f_{i}$. is an approximate dual Banach frame of $\left\{x_{i}\right\}_{i \in I}$ in $X$ with respect to $X_{d}$. Denoting its associated synthesis operator by $U_{n}$, we have

$$
\left\|I_{X^{*}}-U_{n}^{*} V^{*}\right\| \leq\left\|I_{X^{*}}-U^{*} V^{*}\right\|^{n+1} \rightarrow 0 \text { as } n \rightarrow \infty \text {. }
$$

Proof: (i) If $\left\{f_{i}\right\}_{i \in I}$ is an approximate dual Banach frame for $\left\{x_{i}\right\}_{i \in I}$, then the operator $V U$ is a bijection on $X$ and for all $x \in X$ we have 


$$
\begin{aligned}
& x=(V U)(V U)^{-1} x=\sum_{i \in I} f_{i}\left((V U)^{-1} x\right) x_{i} \\
& =\sum_{i \in I}\left(U^{*} V^{*}\right)^{-1}\left(f_{i}\right)(x) x_{i} .
\end{aligned}
$$

Moreover, the inverse of $U V$ can be written as follows:

$$
(V U)^{-1}=\left(I_{X}-\left(I_{X}-V U\right)\right)^{-1}=I_{X}+\sum_{n=1}^{\infty}\left(I_{X}-V U\right)^{n} .
$$

From this the result in (i) follows.

(ii) For any $x \in X$ we have

$$
\begin{aligned}
& \left(I_{X}-V U_{n}\right) x= \\
& x-\sum_{i \in I} f_{n i}(x) x_{i}=x-\sum_{i \in I} \sum_{j=0}\left(I_{X^{*}}-U^{*} V^{*}\right)^{j}\left(f_{i}\right)(x) x_{i} \\
& =x-\sum_{j=0}^{n} V U\left(I_{X}-V U\right)^{j} x=x-\sum_{j=0}^{n}\left(I_{X}-\left(I_{X}-V U\right)\right)\left(I_{X}-V U\right)^{j} x \\
& =\left(I_{X}-V U\right)^{n+1} x .
\end{aligned}
$$

Thus,

$$
\left\|I_{X}-V U_{n}\right\|=\left\|\left(I_{X}-V U\right)^{n+1}\right\| \leq\left\|I_{X}-V U\right\|^{n+1}<1 .
$$

Proposition 3.9: Let $\left\{x_{i}\right\}_{i \in I},\left\{y_{i}\right\}_{i \in I}$ be $X_{d}$-Riesz bases for $X$ with the canonical dual Banach frames $\left\{\tilde{f}_{i}\right\}_{i \in I},\left\{\tilde{g}_{i}\right\}_{i \in I}$ respectively. Then $\left\{\tilde{f}_{i}\right\}_{i \in I}$ is a pseudo-dual Banach frame for $\left\{y_{i}\right\}_{i \in I}$ in $\mathrm{X}$ with respect to $X_{d}$.

Proof: If $V_{1}$ and $V_{2}$ are the synthesis operators of $\left\{x_{i}\right\}_{i \in I},\left\{y_{i}\right\}_{i \in I}$. Then $V_{1}, V_{2}$ are invertible and $V_{1}^{-1}, V_{2}^{-1}$ so are the analysis operators of $\left\{\tilde{f}_{i}\right\}_{i \in I},\left\{\tilde{g}_{i}\right\}_{i \in I}$ respectively. Thus $V_{2} V_{1}^{-1}$ is a bijection on $X$ and for every $x \in X$ we have:

$$
x=\sum_{i \in I} \tilde{f}_{i}(x)\left(V_{1} V_{2}^{-1}\right) y_{i}=\sum_{i \in I} \tilde{f}_{i}\left(\left(V_{1} V_{2}^{-1}\right) x\right) y_{i}
$$

From this the claim follows immediately.

The following result will show that the image of a dual Banach frame under a bounded invertible operators is a pseudo-dual Banach frame.

Theorem 3.10: Let $\left\{f_{i}\right\}_{i \in I},\left\{x_{i}\right\}_{i \in I}$ be $X_{d}$-Bessel and $X_{d^{-}}^{*}$ Bessel sequences for $X, X^{*}$ respectively, and let $\left\{\alpha_{j}\right\}_{j=1}^{N}$ be a set of complex numbers such that $\sum_{j}^{N} \alpha_{j} \neq 0$. Then the following holds:

(i) if $\left\{\left\{f_{i j}\right\}_{i \in I} \mid f_{i j} \in X^{*}, 1 \leq j \leq N\right\}$ is a family of dual Banach frames for $\left\{x_{i}\right\}_{i \in I}$ in $X$ with respect to $X_{d}$ and $\Lambda: X \rightarrow X$ is a invertible operator. Then the sequence $\left\{g_{i}\right\}_{i \in I}$ in $X^{*}$ defined by $g_{i}=\sum_{j=1}^{N} \alpha_{j} \Lambda^{*}\left(f_{i j}\right)$ is a pseudo-dual Banach frame for $\left\{x_{i}\right\}_{i \in I}$ in $X$ with respect to $X_{d}$. (ii) if $\left\{f_{i}\right\}_{i \in I}$ is a dual Banach frame for the family $\left\{\left\{x_{i j}\right\}_{i \in I} \mid x_{i j} \in X, 1 \leq j \leq N\right\}$ in $\mathrm{X}$ with respect to $X_{d}$ and $\Lambda: X \rightarrow X$ is a invertible operator. Then $\left\{f_{i}\right\}_{i \in I}$ is also a pseudo-dual Banach frame for the sequence $\left\{y_{i}\right\}_{i \in I}$ defined by $y_{i}=\sum_{j=1}^{N} \alpha_{j} \Lambda\left(x_{i j}\right)$ in X with respect to $X_{d}$.

Proof: (i) Let $\mathrm{U}$ and $\mathrm{V}$ be the analysis and synthesis operators of $\left\{g_{i}\right\} i \in I$ and $\left\{x_{i}\right\} i \in I$ respectively. Since $\left\{f_{i j}\right\} i \in I$ for all $1 \leq \mathrm{j} \leq \mathrm{N}$ are dual Banach frames for $\left\{x_{i}\right\} i \in I$ in $X$ with respect to $X_{d}$, hence.

hence if $x \in X$ then $x=\sum_{i \in I} f_{i j}(x) x_{i}$. We also have:

$$
\begin{aligned}
V U(x)= & \sum_{i \in I} g_{i}(x) x_{i}=\sum_{i \in I} \sum_{j=1} \alpha_{j} \Lambda^{*}\left(f_{i j}\right)(x) x_{i} \\
& =\sum_{j=1}^{N} \sum_{i \in I} \alpha_{j} f_{i j}(\Lambda x) x_{i}=\left(\sum_{j=1}^{N} \alpha_{j}\right) \Lambda x .
\end{aligned}
$$

(ii) The proof is similar to (i).

Proposition 3.11: Let $\left\{f_{i}\right\}_{i \in I}$ and $\left\{x_{i}\right\}_{i \in I}$ be $X_{d}$-Bessel and $X_{d}^{*}$-Bessel sequences for $X, X^{*}$ and let $\Lambda, \Gamma$ be two invertible operators on $X$. Then a sequence $\left\{f_{i}\right\}_{i \in I}$ is a pseudo-dual Banach frame for $\left\{x_{i}\right\}_{i \in I}$ in $X$ with respect to $X_{d}$ if and only if $\left\{\Gamma^{*} f_{i}\right\}_{i \in I}$ is a pseudo-dual Banach frame for $\left\{\Lambda x_{i}\right\}_{i \in I}$ in $X$ with respect to $X_{d}$.

Proof: Suppose that $U, U_{\Gamma}$ and $V, V_{\Lambda}$ are the analysis and synthesis operators of $\left\{f_{i}\right\}_{i \in I},\left\{\Gamma^{*} f_{i}\right\}_{i \in I}$ and $\left\{x_{i}\right\}_{i \in I},\left\{\Lambda x_{i}\right\}_{i \in I}$ respectively. This claim follows immediately from the fact that for each $x \in X$ we have

$$
V_{\Lambda} U_{\Gamma}(x)=\sum_{i \in I} \Gamma^{*}\left(f_{i}\right)(x) \Lambda x_{i}=\Lambda\left(\sum_{i \in I} f_{i}(\Gamma x) x_{i}\right)=\Lambda V U \Gamma x .
$$

This finishes the proof.

Proposition 3.12: Let $\left\{f_{i}\right\}_{i \in I},\left\{x_{i}\right\}_{i \in I}$ be two $X_{d}$-Bessel and $X_{d}^{*}$-Bessel sequences for $X, X^{*}$ and let $\Lambda, \Gamma$ be invertible operators on $X$. If $\left\{f_{i}\right\}_{i \in I}$ is a dual Banach frame for $\left\{x_{i}\right\}_{i \in I}$ in $X$ with respect to $X_{d}$ then $\left\{\Gamma^{*} f_{i}\right\}_{i \in l}$ is a pseudodual Banach frame for $\left\{\Lambda x_{i}\right\}_{i \in I}$ in $X$ with respect to $X_{d}$.

Proof: The hypotheses imply that $x=\sum_{i \in I} f_{i}(x) x_{i}$. therefore

$$
\sum_{i \in I} \Gamma^{*}\left(f_{i}\right)(x) \Lambda x_{i}=\Lambda\left(\sum_{i \in I} f_{i}(\Gamma x) x_{i}\right)=\Lambda \Gamma x
$$

From this the result follows at once. 
The following we give a method for constructing a family of pseudo-dual Banach frames from a given Banach frame.

Proposition 3.13: Let $\left\{f_{i}\right\}_{i \in I}$ be a pseudo-dual Banach frame for $\left\{x_{i}\right\}_{i \in I}$ in $\mathrm{X}$ with respect to $X_{d}$ with the analysis and synthesis operators $\mathrm{U}, \mathrm{V}$, respectively. If $\alpha, \beta$ are two complex numbers such that $\alpha+\beta=1$. Then the sequence $\left\{g_{i}\right\}_{i \in I}$ defined by $g_{i}=\alpha f_{i}+\beta(V U)^{*}\left(\tilde{f}_{i}\right)$, is a pseudo-dual Banach frame for $\left\{x_{i}\right\}_{i \in I}$ in $X$ with respect to $\mathrm{X}_{\mathrm{d}}$, where $\left(\tilde{f}_{i}\right\}_{i \in I}$ is the canonical dual Banach frame of $\left\{x_{i}\right\}_{i \in I^{\prime}}$.

Proof: For every $x \in X$ we have

$$
\begin{aligned}
& \sum_{i \in I} g_{i}(x) x_{i}=\alpha \sum_{i \in I} f_{i}(x) x_{i}+\beta \sum_{i \in I}(V U)^{*}\left(\tilde{f}_{i}\right)(x) x_{i} \\
& =\alpha V U x+\beta \sum_{i \in I} \tilde{f}_{i}(V U x) x_{i}=(\alpha+\beta) V U x=V U x .
\end{aligned}
$$

The following we give a necessary condition for pseudo-duality of the sum of two pseudo-dual Banach frames from a given Banach frame.

Proposition 3.14: Let $\left\{f_{i}\right\}_{i \in I},\left\{g_{i}\right\}_{i \in I}$ be two pseudodual Banach frames for $\left\{x_{i}\right\}_{i \in I}$ in $\mathrm{X}$ with respect to $\mathrm{X}_{\mathrm{d}}$ with the analysis and synthesis operators $\mathrm{U}_{1}, \mathrm{U}_{2}$ and $V$, respectively. If

$$
\left\|\left(V U_{1}\right)^{-1}|||| V|||| U_{2}\right\|<1,
$$

Then the sequence $\left\{f_{i}+g_{i}\right\}_{i \in I}$ is a pseudo-dual Banach frame for $\left\{x_{i}\right\}_{i \in I}$ in $X$ with respect to $X_{d}$.

PRoof: Since $\left\|(V U 1)^{-1}\right\|\|V\|\left\|U_{2}\right\|<1$ hence the operator $I_{X}+\left(V U_{1}\right)^{-1} V U_{2}$ is invertible which follows that $V\left(U_{1}+U_{2}\right)$ is invertible. Further

$$
\sum_{i \in I}\left(f_{i}+g_{i}\right)(x) x_{i}=\sum_{i \in I} f_{i}(x) x_{i}+\sum_{i \in I} g_{i}(x) x_{i}=V\left(U_{1}+U_{2}\right) x
$$

Therefore $\left\{f_{i}+g_{i}\right\}_{i \in I}$ is a pseudo-dual Banach frame for $\left\{x_{i}\right\}_{i \in I}$.

\section{Acknowledgement}

The authors would like to thank the anonymous reviewers for carefully reading the manuscript and giving useful comments, which has helped to improve the paper.

\section{References}

1. Christensen O, Heil C. Perturbations of Banach frames and atomic decompositions. Math. Nachr. 1997; 185:33-47.

2. Duffin RJ, Schae_er AC. A class of nonharmonic fourier series. Trans. Amer. Math. Soc. 1952; 72(2):341-366.

3. Daubechies I, Grossmann A, Meyer Y. Painless nonorthogonal expansions. J. Math. Phys. 1986; 27:1271-1283.

4. Grochenig K. Describing functions: frames versus atomic decompositions. Monatsh. Math. 1991; 112:1-41.

5. Aldroubi A, Sun Q, Tang W. p-Frames and shift invariant subspaces of $L_{p}$. J. Fourier Anal. Appl. 2001; 7:1-22.

6. Christensen O, Stoeva DT. p-Frames in separable Banach spaces. Adv. Comput. Math. 2003; 18:117-126.

7. Christensen O, Laugesen RS. Approximately dual frames in Hilbert spaces and application to Gabor frames. Sampl Theory Signal Image Process. 2011; 9:77-90.

8. Casazza PG, Han D, Larson D. Frames for Banach spaces. Contemp. Math. 1999; 247:149-182.

9. Zhang H, Zhang J. Frames, Riesz bases, and sampling expansions in Banach spaces via semi-inner products. Appl. Comput Harmon Anal. 2011; 31(1):1-25.

10. Casazza PG, Christensen O, Stoeva DT. Frame expansions in separable Banach spaces. J. Math. Anal. Appl. 2005; 307: 710-723.

11. Christensen O. An introductory course of frames and bases. Birkhauser. 2008; Boston. 